PROCEEDINGS OF THE

AMERICAN MATHEMATICAL SOCIETY

Volume 126, Number 8, August 1998, Pages 2509-2510

S 0002-9939(98)04838-2

\title{
A CORRECTION TO "SMALL REPRESENTATIONS OF FINITE DISTRIBUTIVE LATTICES AS CONGRUENCE LATTICES"
}

\author{
GEORGE GRÄTZER, IVAN RIVAL, AND NEJIB ZAGUIA
}

(Communicated by Lance W. Small)

We would like to thank Ralph Freese for pointing out that Lemma 1 of this paper (these Proceedings 123 (1995), 1959-1961) is stated and proved under the assumption " $\Phi_{1} \prec \Phi_{2}$ in Con $L$ ", but it is used under the assumption " $\Phi_{1} \prec \Phi_{2}$ in the poset of join-irreducible congruences of $L$ ".

We now correctly state and prove Lemma 1 :

Lemma 1. Let $L$ be a finite lattice, and let $v_{i}, u_{i} \in L$ satisfy $v_{i} \prec u_{i}$, for $i=1$, 2. Let $\Phi_{i}=\Theta\left(v_{i}, u_{i}\right)$, for $i=1$, 2. If $\Phi_{1} \prec \Phi_{2}$ in the poset of join-irreducible congruences of $L$, then there is a three-element chain $\left\{e_{1}, h, e_{2}\right\}$ in $L$ such that $\Phi_{i}=\Theta\left(h, e_{i}\right)$, for $i=1,2$, and $e_{1}<h<e_{2}$ or $e_{2}<h<e_{1}$.

Proof. We assume that the reader is familiar with the basic concepts and notations of projectivity in lattices. We follow the notation on pages 129-130 of [1]. Since $v_{1} \equiv u_{1}\left(\Theta\left(v_{2}, u_{2}\right)\right)$ and $v_{1} \prec u_{1}$, by Theorem III.1.2 ibid, there is a sequence of projectivities

$$
u_{2} / v_{2}=y_{1} / x_{1} \nearrow y_{2} / x_{2} \searrow y_{3} / x_{3} \nearrow \cdots \searrow y_{n} / x_{n}=u_{1} / v_{1},
$$

for some natural number $n>1$. Obviously,

$$
\Phi_{2}=\Theta\left(x_{1}, y_{1}\right)=\Theta\left(x_{2}, y_{2}\right) \geq \Theta\left(x_{3}, y_{3}\right) \geq \cdots \geq \Theta\left(x_{n}, y_{n}\right)=\Phi_{1} .
$$

Since $\Phi_{2}>\Phi_{1}$, there is a smallest $i$ satisfying $\Theta\left(x_{i}, y_{i}\right)<\Phi_{2}$; obviously, $3 \leq i$ and $i \leq n$.

Let $i$ be odd, and let $z=x_{i-1} \vee y_{i}$. Then $\Theta\left(x_{i-1}, z\right)=\Theta\left(x_{i}, y_{i}\right)<\Phi_{2}$, but $\Theta\left(x_{i-1}, y_{i-1}\right)=\Phi_{2}$, so $\Theta\left(z, y_{i-1}\right)=\Phi_{2}$. Since $u_{1} \equiv v_{1}\left(\Theta\left(x_{i-1}, z\right)\right)$ and $u_{1} \prec v_{1}$, there are $u, v \in\left[x_{i-1}, z\right], v \prec u$, such that $\Theta\left(u_{1}, v_{1}\right) \leq \Theta(u, v)$. So $\Phi_{1}=\Theta\left(u_{1}, v_{1}\right) \leq$ $\Theta(u, v)<\Phi_{2}$, and $\Theta(u, v)$ is a join-irreducible congruence, hence by the assumption on $\Phi_{1}$ and $\Phi_{2}$, it follows that $\Phi_{1}=\Theta(u, v)$. Hence we can choose $e_{2}=x_{i-1}, h=z$, and $e_{1}=y_{i-1}$.

If $i$ is even, then we proceed dually.

Received by the editors December 1, 1995.

1991 Mathematics Subject Classification. Primary 06B10; Secondary 06D05.

Key words and phrases. Congruence lattice, finite lattice, distributive lattice.

The research of all three authors was supported by the NSERC of Canada.

(C)1998 American Mathematical Society 


\section{REFERENCES}

[1] G. Grätzer, General Lattice Theory, Pure and Applied Mathematics Series, Academic Press, New York, 1978; Mathematische Reihe, Band 52, Birkhäuser Verlag, Basel; Akademie Verlag, Berlin.

Department of Mathematics, University of Manitoba, Winnipeg, Manitoba, Canada R3T $2 \mathrm{~N} 2$

E-mail address: gratzer@cc.umanitoba.ca

Department of Computer Science, University of Ottawa, Ottawa, Ontario, Canada $\mathrm{K} 1 \mathrm{~N} 6 \mathrm{~N} 5$

E-mail address: rival@csi.uottawa.ca

E-mail address: zaguia@csi.uottawa.ca 